\title{
Paleoecología del Holoceno en la Gran Sabana, SE Venezuela: Análisis preliminar de polen y microcarbones en la Laguna Encantada
}

\author{
E. MONTOYA ${ }^{1,2}$, V. RULL ${ }^{1}$, S. NOGUÉ 1,2 \& W. A. DÍAZ ${ }^{3}$ \\ ${ }^{1}$ Institut Botànic de Barcelona (CSIC-ICUB), Psg. Migdia s/n, 08038 Barcelona, Espanya. \\ ${ }^{2}$ Departament de Biologia Animal, Biologia Vegetal i Ecologia, Facultat de Biociències, Universitat Autònoma \\ de Barcelona, Campus Bellaterra, 08193 Barcelona, Espanya. \\ ${ }^{3}$ Universidad Nacional Experimental de Guayana, Centro de Investigaciones Ecológicas de Guayana, Puerto \\ Ordaz, Edo. Bolívar, Venezuela.
}

Autor para correspondencia: E. Montoya (Encarni.Montoya@uab.cat)

Recibido 19 enero 2009; Aceptado 14 mayo 2009

\begin{abstract}
Resumen
La región de la Gran Sabana, al SE de Venezuela, se caracteriza por la presencia de extensas sabanas neotropicales, en un clima cálido y húmedo, que parece más propicio para la existencia de bosques húmedos. Existen dos grandes grupos de hipótesis para explicar esta configuración vegetal: las naturales (cambios climáticos, pobreza de suelos) y las antrópicas (incendios provocados). El presente estudio utiliza el análisis de polen y microcarbones para reconstruir las tendencias paleoambientales, de la vegetación y del régimen de incendios, en la turbera litoral de la Laguna Encantada, durante los últimos 7.500 años. Las sabanas han dominado el paisaje durante todo este tiempo, a pesar de que los incendios locales no afectaron el área hasta hace unos 1.200 años, momento en el cual se establecen las comunidades actuales. Hacia los 4.000 años antes del presente tuvo lugar una expansión de los bosques de galería, causada por cambios climáticos, seguida de una evidente deforestación debida al fuego, que culminó en la situación actual. Estos resultados, junto con estudios anteriores, sugieren que las sabanas actuales de la Gran Sabana son el resultado de la interacción de diversos factores, estando el clima muy asociado a su origen y los fuegos provocados a su gran expansión actual.
\end{abstract}

Palabras clave: Fuego; Mauritia; Microcarbones; Paleoecología; Polen; Sabanas; Venezuela.

\begin{abstract}
Holocene paleoecology of the Gran Sabana, SE Venezuela: preliminary pollen and microcharcoal analysis at Laguna Encantada.- The Gran Sabana region (SE Venezuela) is characterized by extensive neotropical savannas, in a warm and wet climate, apparently more suitable for the existence of rain forests. Two main types of hypotheses have been proposed to explain this situation: natural factors, such as climate changes or soil poverty, and burning by humans. This study analyzes the pollen and charcoal content of a 7,500 years old peat bog, at the shore of Laguna Encantada. The savanna landscape has dominated the area during the whole interval, but local fires did not appear until around 1,200 years ago, when the present communities began to establish. An expansion of gallery forests, likely due to a climatic shift, has been recorded around 4,000 years ago, followed by a deforestation triggered by fire, leading to present-day situation. These results, together with former similar studies, suggest that the present Gran Sabana landscape resulted from the interaction of diverse factors, being climate changes more related to its origin and human induced fires more linked to the recent expansion.
\end{abstract}

Key words: Fire; Mauritia; Microcharcoal; Palaeoecology; Pollen; Savannas; Venezuela. 


\section{INTRODUCCIÓN}

El estudio de restos biológicos (polen, esporas, algas, hongos, etc.), como indicadores de situaciones pasadas en sedimentos lacustres, turberas, etc., ha despertado un creciente interés desde la segunda mitad del siglo pasado (Birks \& Birks, 1980). Muchos de estos estudios paleoecológicos están basados en análisis palinológicos. La identificación de taxones vegetales a partir de los granos de polen y esporas no sólo permite reconstruir las comunidades vegetales del pasado, sino que contribuye a establecer la evolución climática de una determinada región (Faegri et al., 1989). Otro de los indicadores frecuentemente utilizados en paleoecología son los microcarbones. Estas partículas se encuentran en el sedimento como consecuencia de los incendios que tienen lugar en la zona de influencia. Se trata, en su mayoría, de restos de cenizas, generalmente procedentes de material vegetal quemado. El uso de carbones macro- y microscópicos como apoyo a estudios paleoecológicos ha ido en aumento durante los últimos 20 años. Su estudio, junto con el de polen y esporas, nos proporciona información acerca del clima, la vegetación, el régimen de incendios y las actividades humanas en el pasado (Kangur, 2002; Hammond et al., 2006; Enache \& Cumming, 2007; Duffin et al., 2008).

El presente estudio se desarrolla en una turbera de la Gran Sabana (GS), situada en el SE de Venezuela (Fig. 1). Las sabanas han despertado gran interés por su característica distribución geográfica y por la gran extensión que ocupan (aproximadamente 3 millones de $\mathrm{km}^{2}$ sólo en el trópico americano [Huber, 1987]). Existen numerosos estudios paleoecológicos realizados en sabanas neotropicales o zonas de mosaico bosque-sabana, cuyo propósito es determinar el origen de este tipo de vegetación e inferir los mecanismos que han actuado para la continuidad en el tiempo de la misma (por ejemplo, Wymstra \& Van der Hammen, 1966; Servant et al., 1993; Van der Hammen \& Absy, 1994; Bush et al., 2004). Un aspecto importante en el estudio del origen y extensión de estas sabanas ha sido su relación con el fuego, ya que estas comunidades sufren una alta presión por incendios (Beerling \& Osborne, 2006; Ramírez et al., 2007). En la GS, por ejemplo, se registran más de 10.000 incendios de origen antrópico cada año (Huber, 1995b), causados principalmente por agricultores, indígenas y mineros. Diversos estu- dios indican que, teniendo en cuenta los regímenes de temperatura y precipitación de la GS (ver apartado de área de estudio), esta región posee un bioclima típico de bosque montano perennifolio (Huber, 1995c; Ramírez et al., 2007). Sin embargo, su vegetación actual se caracteriza por vastas extensiones de sabana y mosaicos bosque-sabana (Baruch, 2005), lo que ha llevado a plantearse las posibles causas de la presencia y extensión de esta formación vegetal. Las principales causas propuestas hasta ahora son:

- Las condiciones edáficas, sobre todo la pobreza en nutrientes y las relaciones hídricas (Fölster, 1986; Fölster et al., 2001).

- Que las sabanas actuales sean un relicto de extensiones de esta formación en épocas pasadas, como durante el último máximo glaciar ( $c a$. 20.000 años) (Eden, 1974).

- La acción directa del fuego (Dezzeo et al., 2004).

- La expansión de sabana a partir de núcleos aislados preexistentes debido a la presión humana, principalmente a través de los incendios (Rull, 1999; Huber, 2006).

Los trabajos efectuados hasta el momento han puesto de manifiesto la baja resiliencia de los ecosistemas forestales de la GS. Se ha manifestado el escaso o nulo poder de recuperación del bosque con posterioridad a eventos de deforestación, a corto y largo plazo, bien sea por causas naturales o antrópicas (Fölster, 1992; Rull, 1992, 1999; Fölster et al., 2001; Dezzeo \& Chacón, 2005; Dezzeo \& Chacón, 2006). La capacidad de regeneración del bosque estaría limitada por la influencia de los factores edáficos y la rápida colonización por plantas herbáceas a partir de sabanas cercanas, así como por la alta frecuencia de incendios. El resultado de este conjunto de factores es una sucesión secundaria que, a largo plazo, termina en la instalación de sabanas inarboladas o morichales (comunidades monoespecíficas de palmeras dominadas por Mauritia flexuosa, frecuentes en suelos mal drenados como los alrededores de las lagunas y a lo largo de los ríos), según sean las condiciones de humedad (Rull, 1992).

En la GS y sabanas neotropicales cercanas, el Holoceno (los últimos 11.500 años) constituye una época de fluctuaciones ambientales, lo que ha afectado a la vegetación del entorno (Rull et al., en prensa). El inicio del Holoceno se caracterizó, en la Gran Sabana, por un aumento en las tempera- 
turas medias de $2-3^{\circ} \mathrm{C}$ posterior al último máximo glacial, y que muy probablemente repercutió en la sustitución de la vegetación dominante hasta ese momento, un bosque mesófilo húmedo, por una sabana inarbolada (Rull, 2007). Esta interpretación discrepa con la hipótesis propuesta por Eden (1974), que afirma que la vegetación de sabana constituye un relicto del último máximo glacial. Otros estudios ilustran como las oscilaciones ambientales y el fuego han influido fuertemente en el paisaje vegetal en los últimos milenios (véase por ejemplo, Rull, 1991). En general, las consecuencias de las fases de clima más seco y las de mayor incidencia de fuegos han sido similares, manifestándose en ambos casos en una reducción de los bosques y expansión de las sabanas (Rull, 1992). Prácticamente toda la información paleoecológica de que se dispone para la región corresponde a los últimos 4.000 años, fecha desde la cual, la incidencia de fuegos ha aumentado considerablemente (Rull, 1991; Fölster, 1992). En estos estudios, se ha observado que después de cada perturbación causada indistintamente por el clima o el fuego, se desencadenan procesos de sucesión secundaria que terminan en el establecimiento de sabanas, con o sin morichales. El retorno de condiciones húmedas después de las fases climáticas secas o los incendios no ha conducido a la regeneración de los bosques, sino que en su lugar, se han establecido comunidades casi monoespecíficas de Mauritia flexuosa (Rull, 1992, 1999). Estos morichales han aumentado en los últimos milenios y se ha propuesto que están en expansión desde la última glaciación, favorecidos por el fuego (Rull, 1998a).

El propósito de este trabajo es reconstruir la vegetación y sus cambios a escala de milenios en una secuencia continua de 7.500 años de antigüedad, con especial interés en determinar las causas del establecimiento de la comunidad actual de sabanas con morichales en la zona de estudio. Se pretende reconstruir la dinámica de la vegetación durante el Holoceno y analizar el papel del clima y del fuego, con el fin de evaluar las hipótesis sobre el origen y el mantenimiento de la actual Gran Sabana.

\section{Área de estudio}

La Gran Sabana es una extensa región de unos $18.000 \mathrm{~km}^{2}$ situada al SE de Venezuela $(4,6089$ 6,6331 N y 61,0679-74,0478 W, Fig. 1), aunque las sabanas continúan más allá de estas fronteras, penetrando en Guyana y Brasil, con una extensión total de aproximadamente $30.000 \mathrm{~km}^{2}$. Esta región se extiende entre los 750 y $1.450 \mathrm{~m}$ de altitud, con un gradiente descendente de $\mathrm{N}$ a S. Desde el punto de vista fisiográfico, forma parte de las llamadas "tierras medias" o uplands que, en la región de Guayana, se sitúan entre 500 y 1.500 m de altitud (Huber, 1995a). Desde el punto de vista geológico, la GS se encuentra enteramente sobre el sector nororiental del Escudo de Guayana, uno de los núcleos terrestres más antiguos del continente americano. Dependiendo de la geología predominante, la geomorfología de la zona varía notablemente, desde llanuras onduladas asociadas a sustratos de arenisca, a colinas y lomas bajas sobre diabasa (Huber \& Febres, 2000). Dentro de las seis superficies de erosión que se han descrito para el Escudo de Guayana, la GS se encuentra en su mayoría incluida en la de Wonkén, de 900 a $1.200 \mathrm{~m}$ de altitud (Briceño \& Schubert, 1990).

El clima de la región es de tipo submesotérmico, con una temperatura media anual de $18-20^{\circ} \mathrm{C}$. El régimen pluviométrico es de tipo ombrófilo (2.500$4.000 \mathrm{~mm}$ anuales), existiendo notables variaciones locales. La distribución anual de la precipitación está condicionada por la variación estacional de la Zona de Convergencia Intertropical (ITCZ), lo que se traduce en una alternancia anual de las precipitaciones que determina la existencia de una estación lluviosa entre abril y noviembre, y otra más seca entre diciembre y marzo. Ambos parámetros climáticos muestran un claro gradiente en sentido N-S, siendo ascendente para la temperatura y descendente para la precipitación (Galán, 1984; Huber \& Febres, 2000). Según Huber (1995c), la vegetación de la GS se puede dividir en cuatro tipos principales: 1) formación boscosa, en la cual se pueden diferenciar bosques de tipo montano y bosques de galería; 2) formación arbustiva, con las comunidades de mayor diversidad en la zona (Huber, 1986; Huber, 1995c); 3) formación herbácea, en la que se pueden distinguir los herbazales gramíneos o sabanas, y los herbazales no gramíneos o praderas; y 4) formación pionera, caracterizada por una vegetación dispersa y una alta especialización de arbustos y herbáceas. Dentro de las sabanas, se pueden distinguir a su vez tres tipos (Huber, 1995b): 1) Sabanas abiertas o lisas, el tipo más extendido, llamadas también sabanas inarboladas. En estas sabanas existe un estrato herbáceo de hasta 
$1 \mathrm{~m}$ de alto, de densidad variable. Son comunidades pobres en especies (20-30 especies), donde pueden aparecer elementos leñosos (arbustos o sufrútices) aislados, que no superan la altura alcanzada por las plantas herbáceas. 2) Sabanas con morichales, estacionalmente inundadas, donde se forma un denso estrato herbáceo de hasta $2 \mathrm{~m}$ de alto con parches más o menos dispersos de morichales. 3) Sabanas arbustivas, en suelos extremadamente pobres en nutrientes y ácidos. El estrato herbáceo aparece disperso e irregular, y dominan taxones de la familia Cyperaceae.

\section{MATERIAL Y MÉTODOS}

La turbera objeto de este estudio se encuentra en el margen de la Laguna Encantada $(4,7110 \mathrm{~N}$ 61,0821 W; $867 \mathrm{~m}$ altitud), dentro de la finca privada denominada "Hato Santa Teresa", cercana a la ciudad de Santa Elena de Uairén (Fig. 1). Esta ciudad se ubica en la zona sur de la GS a unos $910 \mathrm{~m}$ de altitud. Sus registros pluviométricos señalan una precipitación total anual de 1.700 mm (Galán, 1984). La laguna se encuentra rodeada por una extensa sabana abierta que, en sus orillas, se transforma en una turbera con individuos dispersos de Mauritia flexuosa. E1 sondeo PATAM4_D07 se realizó en una de estas turberas en enero de 2007, utilizando una sonda rusa (Jowsey, 1966), con la que se obtuvo una secuencia total de $392 \mathrm{~cm}$ de profundidad. En ella se realizaron un total de 10 dataciones de ${ }^{14} \mathrm{C}$ mediante la técnica de espectometría de aceleración de masas (AMS), sobre macrorrestos vegetales o extractos polínicos, en los laboratorios UCI (Laboratorio de Radiocarbono de la Universidad de California, USA) y Beta Analytic (Laboratorio comercial, USA). La calibración de estas dataciones se llevó a cabo con el software CALIB 5.0.2 y la base de datos IntCa104.14c (http://calib.qub. ac.uk./calib/, última consulta en mayo del 2008). Las edades obtenidas se expresaron finalmente como años calibrados antes del presente o cal BP (Wolff, 2007). El modelo de profundidad-edad del sedimento se realizó mediante el software $\mathrm{R}$ (http://www.r-project.org, última consulta en mayo del 2008), utilizando el paquete de datos age.R (http://chrono.qub.ac.uk/blaauw/, última consulta en mayo de 2008). El modelo se basó en la interpolación lineal de las edades obtenidas en la calibración (Blaauw et al., en prep.). Para el presente análisis polínico preliminar de la secuencia se analizaron 25 muestras con una separación de entre $15-20 \mathrm{~cm}$ entre cada una. El tratamiento de laboratorio consistió en sucesivas digestiones con $\mathrm{KOH}, \mathrm{HCl}, \mathrm{HF}$ y mezcla de acetólisis (anhídrido acético y ácido sulfúrico en proporción 9:1) (Faegri et al., 1989; Moore et al., 1991). El medio de montaje empleado fue aceite de silicona, por lo que las muestras pasaron por un proceso previo de deshidratación, por imbibición secuencial en etanol y butanol terciario. Antes del tratamiento, se introdujeron tabletas con esporas de Lycopodium (batch 124.961, con $12.542 \pm 2.081$ esporas por comprimido), según Benninghoff (1962) y Stockmarr (1971). Para la identificación de palinomorfos se utilizaron las siguientes referencias: Hooghiemstra (1984); Moore et al. (1991); Roubik \& Moreno (1991); Tryon \& Lugardon (1991); Herrera \& Urrego (1996); Rull \& Vegas-Vilarrúbia (1997); Rull (1998b); Colinvaux et al. (1999) y Rull (2003). El conteo se hizo siguiendo las relaciones de calidad/esfuerzo propuestas en Rull (1987). La suma de polen incluye todos los taxones polínicos identificados, excepto las especies acuáticas. No se incluyeron en el diagrama los taxones con proporciones inferiores al $0,1 \%$. Para la construcción y zonación de los diagramas polínicos, se utilizó el software PSIMPOLL 4.26 (http://www. chrono.qub.ac.uk/psimpoll/psimpoll.html, última consulta realizada en marzo del 2008) y los criterios de Bennett (1996). La interpretación de los espectros polínicos se basó en estudios previos de sedimentación polínica actual en relación con la vegetación (Rull, 1991). Los cálculos del índice de diversidad de Shannon $\left(\mathrm{H}^{\prime}\right)$, la equitabilidad (E) y la riqueza de especies (S) (Pielou, 1966; Hurlbert, 1971), se llevaron a cabo mediante el programa MVSP versión 3.13. El conteo de microcarbones se efectuó en las mismas preparaciones destinadas al análisis palinológico. Se contaron las partículas mayores de $5 \mu \mathrm{m}$ y se establecieron tres grupos diferentes dependientes del tamaño, siguiendo la clasificación para la Gran Sabana de Rull (1999):

1. Microcarbones menores de $100 \mu \mathrm{m}$ (Tipo I): como indicadores de fuegos regionales, al ser fácilmente transportados por el viento. 
2. Microcarbones comprendidos entre $100 \mathrm{y}$ $500 \mu \mathrm{m}$ (Tipo II): indicadores de incendios locales o cercanos a la zona de sondeo.

3. Microcarbones mayores de $500 \mu \mathrm{m}$ (Tipo III): indicadores de incendios locales e intensos.

\section{RESULTADOS}

\section{Cronología}

De las 10 dataciones realizadas, se utilizaron 7 para establecer el modelo de profundidad-edad (Tabla 1, Fig. 2). Las otras 3 dataciones fueron descartadas por presentar edades incongruentes, debido probablemente a contaminación por material más joven procedente de niveles superiores El modelo sedimentario muestra un testigo continuo con distintas velocidades de acumulación, en el que se diferencian tres zonas principales (Fig. 2):

1. La parte más profunda $(390-310 \mathrm{~cm}, 7.500-$ 7.095 años cal BP), con una rápida velocidad de acumulación $(0,223 \mathrm{~cm} /$ año$)$.

2. El tramo intermedio $(310-240 \mathrm{~cm}, 7.095-$ 3.400 años cal BP), con una velocidad marcadamente inferior $(0,0178 \mathrm{~cm} /$ año$)$.

3. El tramo superior $(240-10 \mathrm{~cm}, 3.400$ años cal BP al presente), con una tasa de acumulación intermedia $(0,069 \mathrm{~cm} / \mathrm{año})$.

La naturaleza y composición del sedimento no es homogénea a lo largo de la secuencia (Fig. 2). El testigo comienza siendo una turba oscura con contenido arcilloso $(391,5-384,5 \mathrm{~cm})$. Seguidamente, se observa un intervalo $(384,5-306 \mathrm{~cm}) \mathrm{de}$ arcillas grisáceas con intercalaciones de arena. A continuación (306-200 cm), comienza de nuevo un sedimento turboso, el cual contiene al inicio una porción arcillosa oscura. La parte más reciente del testigo $(200-10 \mathrm{~cm})$, se caracteriza por una turba oscura, poco compactada y con elevado contenido en agua. Se consideró la posibilidad de un hiatus a $300 \mathrm{~cm}$, en el contacto entre arcilla y turba, pero se aceptó el modelo de sedimentación continua por ser el más significativo estadísticamente.

\section{Polen y esporas}

El diagrama polínico (Fig. 3) se subdividió en tres zonas polínicas que se describen a continuación. La muestra de la base de la secuencia se describe

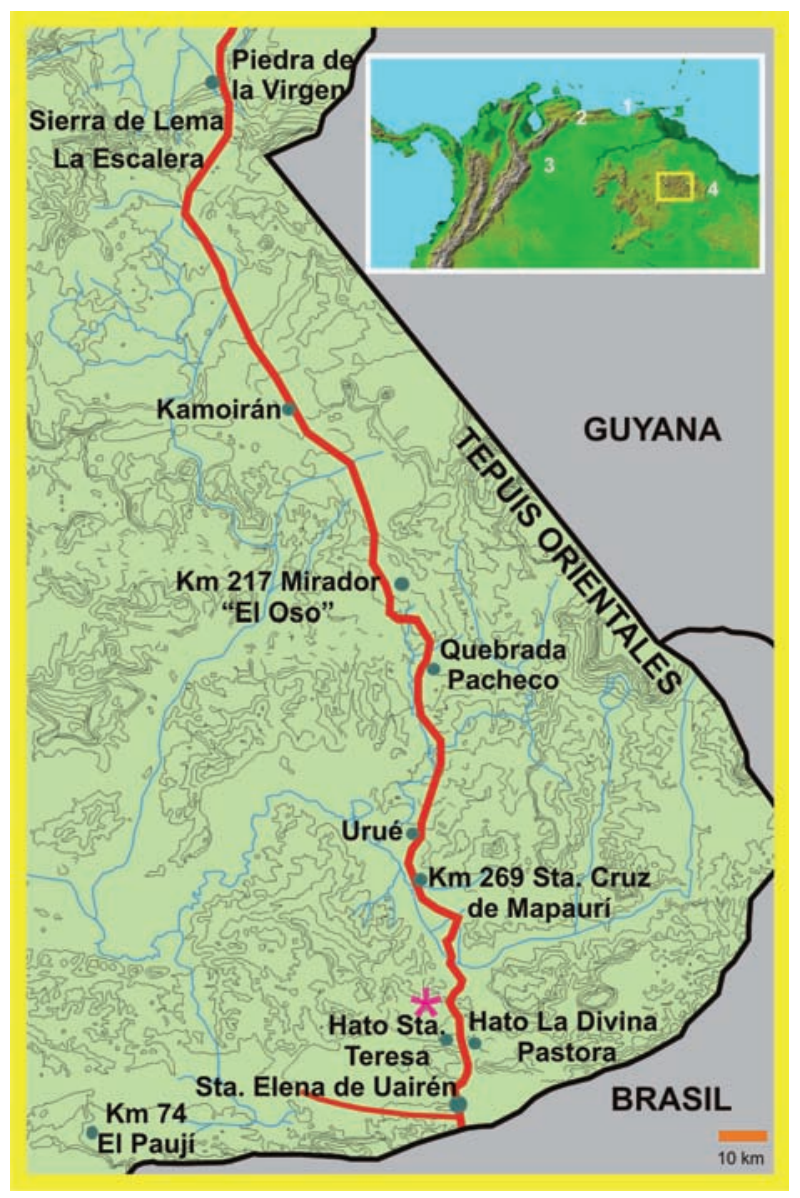

Figura 1. Localización del área de estudio. Recuadro externo: Mapa ampliado de la Gran Sabana, donde el asterisco señala el lugar del sondeo. Recuadro interno: Imagen de radar del norte de Sudamérica (NASA/ JPL-Caltech). El rectángulo amarillo señala la región de la Gran Sabana, mientras que los diferentes números muestran regiones bajas donde se han llevado a cabo los estudios paleoecológicos mencionados en el texto, siendo: (1) Cuenca de Cariaco (Venezuela); (2) Lago Valencia (Venezuela); (3) Llanos Orientales de Colombia; y (4) Sabanas de Guyana.

aisladamente pese no a no ser significativamente distinta, debido a las diferencias sedimentológicas y palinológicas que presenta con el resto de la secuencia. La distancia entre muestras $(15-20 \mathrm{~cm})$ supone una diferencia de edad de entre 280 y 350 años, por lo que la resolución de esta reconstrucción es a escala de milenios.

\section{Muestra 81 (392-389 cm, 1 muestra)}

Caracterizada por su alto contenido orgánico y por sus elevados porcentajes de gramíneas y otros 
Tabla 1. Resultados de las dataciones (Acrónimo de los laboratorios responsables: UCI: Universidad de California; y Beta: Laboratorio comercial Beta Analytic Inc.). Las muestras utilizadas para el modelo de profundidad-edad se han marcado con un asterisco.

\begin{tabular}{|c|c|c|c|c|c|}
\hline $\mathbf{N}^{0}$ Laboratorio & Muestra & $\begin{array}{l}\text { Profundidad } \\
(\mathrm{cm})\end{array}$ & Tipo muestra & $\begin{array}{c}\text { Edad } \\
\left(\text { años }{ }^{14} \mathrm{C}\right. \\
\text { BP) }\end{array}$ & $\begin{array}{c}\text { Edad } \\
\text { (años cal BP) } \\
\operatorname{con} 2 \sigma\end{array}$ \\
\hline UCI $-43721 *$ & PATAM4_D07/10 & $55-60$ & Macrofósil & $655 \pm 35$ & $555-609$ \\
\hline UCI $-43694 *$ & PATAM4_D07/22 & $115-120$ & Macrofósil & $1.235 \pm 20$ & $1.079-1.188$ \\
\hline $\mathrm{UCI}-43695$ & PATAM4_D07/34 & $175-180$ & Macrofósil & $790 \pm 20$ & $680-732$ \\
\hline Beta $-242280 *$ & PATAM4_D07/35 & $180-185$ & Macrofósil & $2.090 \pm 40$ & $1.949-2.152$ \\
\hline Beta $-243846 *$ & PATAM4_D07/46 & $235-240$ & Extracto polínico & $3.140 \pm 40$ & $3.335-3.404$ \\
\hline UCI - 43696 & PATAM4_D07/47 & $240-245$ & Macrofósil & $1.930 \pm 20$ & $1.825-1.903$ \\
\hline UCI - 43697 & PATAM4_D07/59 & $300-303$ & Macrofósil & $445 \pm 20$ & $487-524$ \\
\hline Beta $-242281 *$ & PATAM4_D07/60 & $303-306$ & Extracto polínico & $6.170 \pm 40$ & $6.949-7.169$ \\
\hline Beta $-242282 *$ & PATAM4_D07/74 & $362-367$ & Extracto polínico & $6.530 \pm 40$ & $7.414-7.511$ \\
\hline UCI $-37498 *$ & PATAM4_D07/81 & $388-393$ & Macrofósil & $6.530 \pm 20$ & $7.422-7.476$ \\
\hline
\end{tabular}

elementos herbáceos como Xyris, así como algunos taxones leñosos (Byrsonima, Miconia) y, en una menor proporción, Urticales. El resto de taxones arbóreos y arbustivos se encuentran en baja proporción o están ausentes.

\section{Zona SM-I (389-198 cm, 12 muestras)}

En general, se aprecia un aumento de elementos forestales y algas con respecto a la muestra anterior. Entre los taxones leñosos destacan Brosimum y otras Urticales, Weinmannia, Malpighiaceae y Miconia. Estos elementos se alternan en abundancia con las gramíneas en la mitad inferior de la zona, mientras que en la mitad superior aumentan notablemente, coincidiendo con una mayor abundancia de algas. En esta mitad superior, también aumentan otros elementos forestales como Schefflera, Acalypha y Alchornea. Mauritia está ausente en esta parte de la secuencia.

\section{Zona SM-II (198-120,5 cm, 5 muestras)}

Caracterizada por una disminución de la mayoría de taxones leñosos (Acalypha, Alchornea, Byrsonima, Schefflera, Urticales, Malpighiaceae, Miconia y Myrsine). Los taxones herbáceos sufren un aumento evidente, sobre todo las gramíneas y Xyris, y en menor medida Eriocaulaceae. Las esporas también aumentan, sobre todo los triletes psilados. En esta zona se registran las primeras apariciones de Mauritia, aunque en una baja proporción. Las algas mantienen niveles similares o ligeramente inferiores a la parte final de la anterior zona.

\section{Zona SM-III (120,5-10 cm, 7 muestras)}

La mayoría de taxones leñosos y esporas de pteridófitos desaparecen casi totalmente a excepción de Mauritia, que experimenta un notable y súbito aumento hasta convertirse en el taxón subdominante después de las gramíneas. Las gramíneas aumentan de nuevo en la primera parte de esta zona, y otras herbáceas como Eriocaulaceae y Xyris se mantienen en proporciones similares a la zona anterior. Las algas disminuyen en esta zona.

\section{Algas \\ Las algas y los macrófitos acuáticos se representan en un diagrama separado para ilustrar las variacio- nes del medio acuático (Fig. 4). Algunos taxones muestran una presencia más o menos continua a lo largo del sedimento (Zygnemataceae, Cyperaceae y Sagittaria), mientras que otros aparecieron sólo de manera puntual en un tramo de la secuencia (Botryococcus, Utricularia y Spirogyra). Existe un marcado pico de Zygnemataceae a $270 \mathrm{~cm}$, en la Zona SM-I, simultáneo con la aparición de Botryococcus, que no está acompañado por cambios en las plantas acuáticas. Sin embargo, otro aumento de las algas, registrado en la mi- tad superior de la Zona SM-II, sí que coincide con picos de Cyperaceae y Sagittaria. En la Zona}




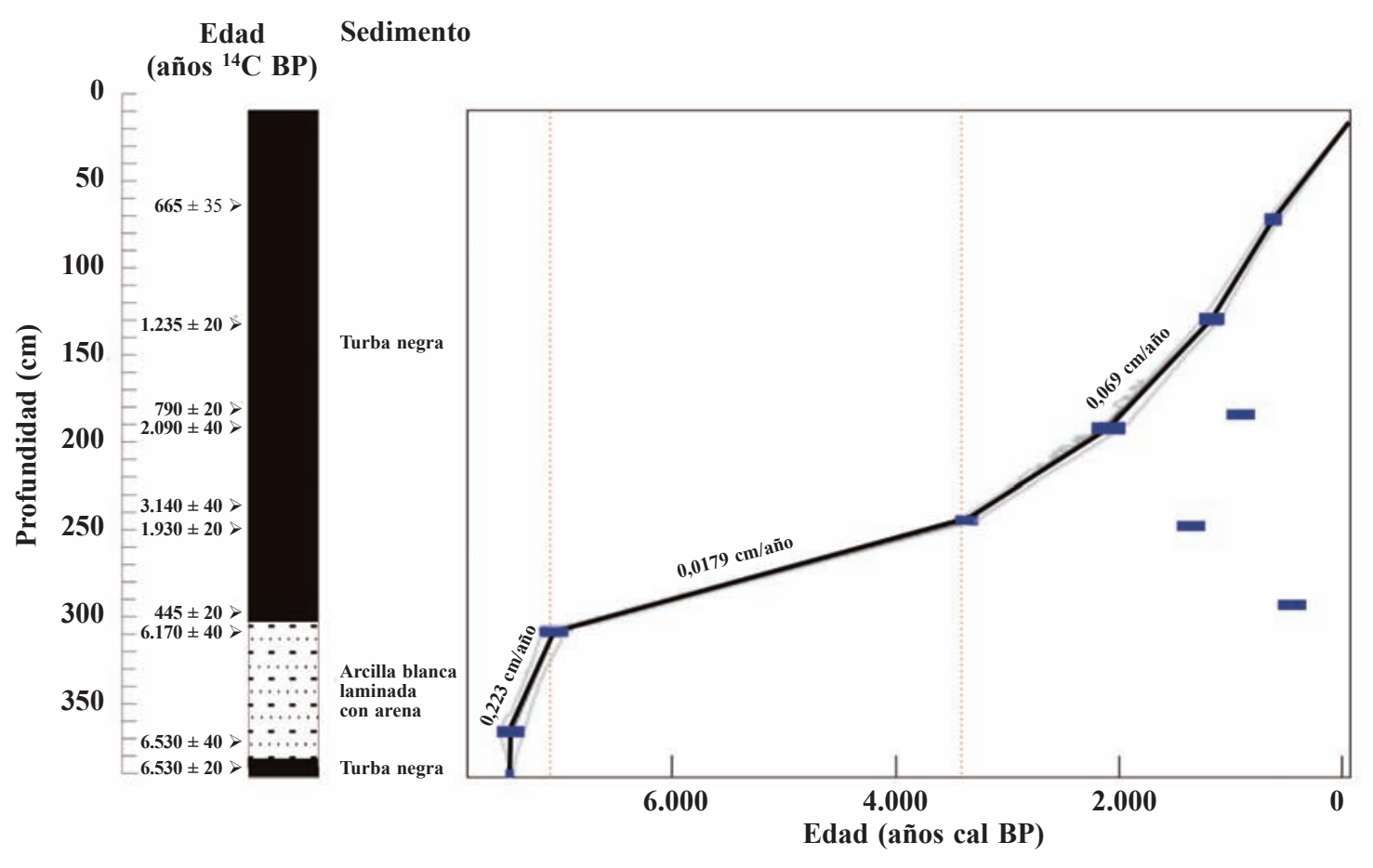

Figura 2. Columna estratigráfica del testigo analizado, con las dataciones realizadas (edades no calibradas) y la descripción de la columna sedimentaria, junto con el modelo de profundidad-edad, con las tasas de sedimentación de cada intervalo. Los cuadros azules representan las dataciones realizadas con sus respectivos errores.

SM-III, se aprecia una disminución general de los taxones acuáticos mayoritarios.

\section{Microcarbones}

La presencia de microcarbones es continua durante toda la secuencia, apareciendo con menores concentraciones en la Zona SM-I y primera mitad de la SM-II. En la segunda mitad de la Zona SM-II aumentan de forma muy acusada, manteniéndose en valores similares (aunque con grandes fluctuaciones) en la Zona SM-III (Fig. 5). En la Zona SM-I, los microcarbones de menor tamaño (I) son los más abundantes, mientras que los de tamaño intermedio (II) están presentes en muy baja proporción y la clase III está ausente. En la Zona SM-II, aumentan ligeramente los intermedios y los menores se mantienen similares, a excepción de la última muestra en la que experimentan un gran aumento. Por último, en SM-III, se produce el mayor aumento en los microcarbones, debido principalmente a los de menor tamaño, aunque los intermedios aumentan también su concentración. Los microcarbones de mayor tamaño (tipo III) se mantienen constantes en cantidades insignificantes durante toda la secuencia.

\section{Riqueza y diversidad}

El índice de diversidad de Shannon oscila en la mitad inferior de SM-I y se estabiliza en valores altos en la parte superior de esta zona (Fig. 5). En la Zona SM-II, este índice sufre una progresiva disminución, que sigue hasta la Zona SM-III, donde alcanza su mínimo. El valor de equitabilidad también oscila en la mitad inferior de SM-I y posteriormente se estabiliza hasta experimentar una ligera disminución en SM-II y un mínimo en SM-III. La riqueza de taxones se mantiene en un rango similar desde la base del diagrama hasta SM-III, donde se evidencia una ligera disminución. En general, las tres curvas presentan una tendencia descendente a lo largo de la secuencia, que se acentúa en SM-III. 


\section{INTERPRETACIÓN Y DISCUSIÓN}

La secuencia estudiada refleja la dinámica de la vegetación alrededor de la Laguna Encantada en los últimos 7.500 años. Los cambios observados se pueden interpretar en términos de variaciones ambientales, sobre todo en el balance hídrico (P/E), entendido como la relación entre la precipitación (P) y la evapotranspiración (E), y del régimen de incendios. La interpretación paleoecológica resultante se resume en la Fig. 6 y se describe a continuación, comparándola con otros estudios de áreas neotropicales vecinas.

\section{Reconstrucción paleoambiental y de la vegetación}

\subsection{0-7.450 cal BP (Muestra 81)}

La abundancia de taxones herbáceos junto a la presencia, en baja proporción, de algunos elementos leñosos se interpreta como indicativa de una formación de sabana herbáceo-fruticosa o de sabana mixta. La baja proporción de esporas y algas sugiere un balance hídrico similar al actual o ligeramente más seco. Este tipo de vegetación, por lo demás pobre en taxones, es consistente con climas menos pluviosos o más estacionales que los actuales, como proponen Behling \& Hooghiemstra (1998b) para regiones cercanas. Trabajos realizados en zonas similares como las sabanas de los Llanos de Colombia o el Lago Valencia (Fig. 1), también ponen de manifiesto condiciones más secas que las actuales durante el Holoceno Temprano, cuando las formaciones de sabana predominaban sobre las formaciones boscosas en el norte de Sudamérica (Wymstra \& Van der Hammen, 1966; Leyden, 1985; Behling \& Hooghiemstra, 1998b; Berrío et al., 2002).

\subsection{0-2.470 cal AP (Zona SM-I)}

El cambio sedimentológico desde la turba orgánica de la muestra anterior a las arcillas con intercalaciones de arena de la base de este intervalo, indica la presencia de un curso de agua de baja energía, lo que sugiere un aumento en el balance hídrico. En esta parte del testigo, la velocidad de sedimentación es muy rápida, lo cual es consistente con una mayor tasa de aporte de sedimentos clásticos alóctonos, debida a un aumento de la escorrentía. El aumento de taxones leñosos en este mismo intervalo se puede interpretar como una mayor extensión de los bosques de galería. Esta situación se había mantenido en una primera etapa, hasta los 7.180 años cal BP. En el segundo tramo de la zona (7.180-2.470 cal BP), la tasa de sedimentación es muy inferior (Fig. 2) y el sedimento vuelve a caracterizarse por un alto porcentaje de la fracción orgánica (autóctona), aunque con contenido en arcillas, lo que podría estar ocasionado por la formación de una laguna similar a la actual. El aumento de los elementos forestales (que indica mayor extensión o proximidad de los bosques) y el aumento de los taxones acuáticos alrededor de $c a .4 .000$ años cal BP, apoyan la interpretación de un mayor balance hídrico, que estaría de acuerdo con la hipótesis de Marchant \& Hooghiemstra (2004), del predominio de climas húmedos a escala del trópico sudamericano, hacia los 4.000 años cal BP. El final del Holoceno Temprano y comienzos del Holoceno Medio parece haberse caracterizado, en las zonas bajas del Norte de Sudamérica, por un aumento del balance hídrico, paralelamente con un aumento en la extensión de las formaciones forestales (Berrío et al., 2002; Marchant \& Hooghiemstra, 2004; Behling \& Hooghiemstra, 1998b). En el Lago Valencia, esta fase corresponde a niveles lacustres altos (Curtis et al., 1999), mientras que en la cuenca de Cariaco (Fig. 1), las evidencias isotópicas y geoquímicas apuntan a un aumento de la precipitación hacia el mismo periodo, que se relacionan con un desplazamiento hacia el norte de la Zona de Convergencia Intertropical (ITCZ), que regula el régimen de precipitaciones (Haug et al., 2001).

\subsection{0-1.220 cal BP (Zona SM-II)}

La disminución de los elementos arbóreos y las algas, junto con la gran dominancia de los taxones herbáceos se interpreta como una expansión de las sabanas y una retracción de los bosques. Dado que no se observa aumento de la concentración de microcarbones, estos cambios vegetales se interpretan como de origen climático. Todo ello sugiere una disminución del balance hídrico y niveles lacustres más bajos. Hacia la mitad de esta zona, los primeros individuos de Mauritia llegaron a la Laguna Encantada, pero sin formar todavía morichales. La existencia de condiciones más secas a nivel regional se manifiesta en un descenso de los niveles lacustres en 


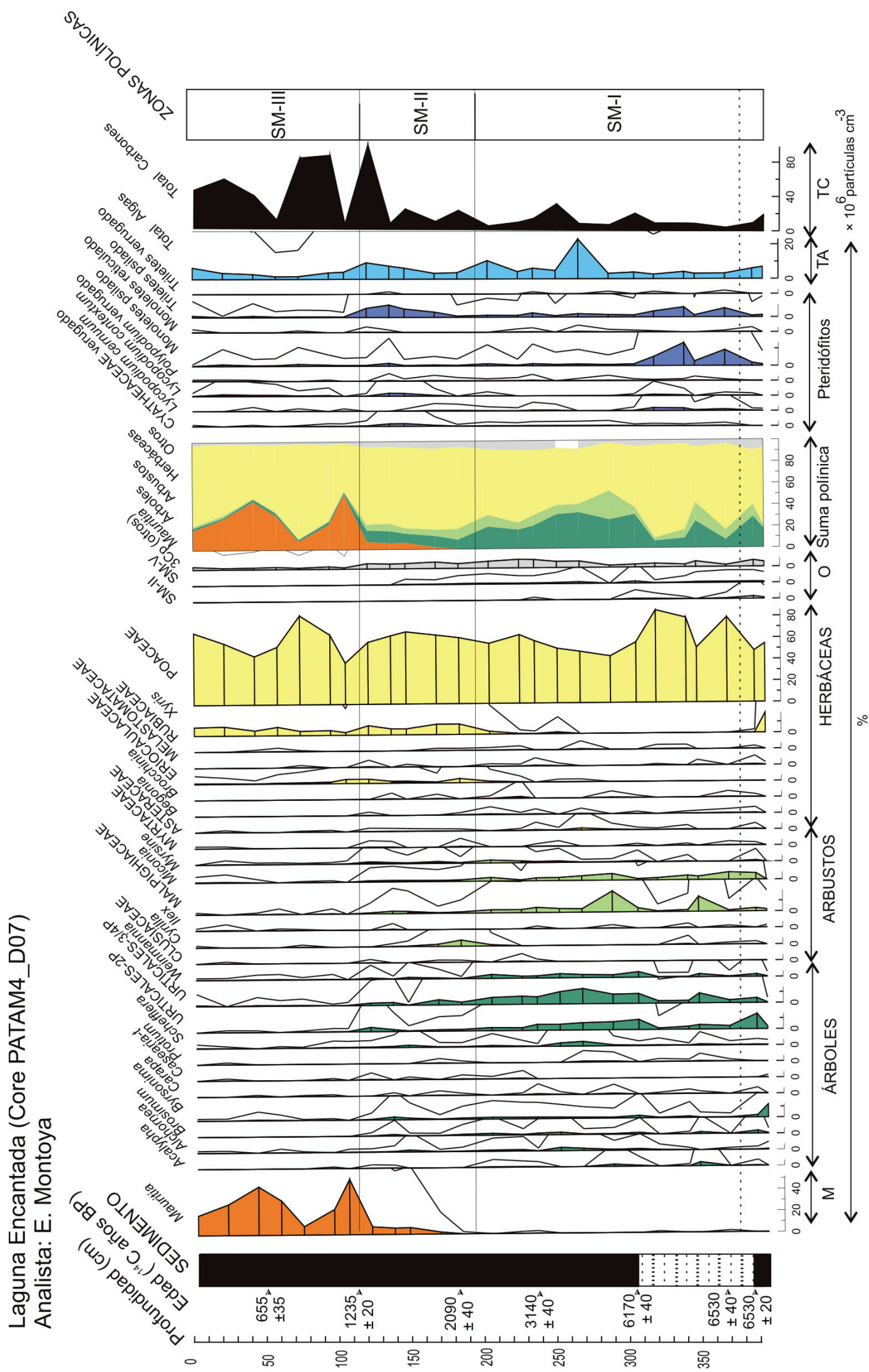

Figura 3. Diagrama polínico de la secuencia, expresado en porcentaje con respecto a la suma polínica. M, Mauritia; O, otros; TA, total de algas; TC, total de carbones; SM-II y SM-V, morfotipos polínicos indeterminados; $3 \mathrm{Cp}$, polen tricolporado no identificado. 
el Lago Valencia (Leyden, 1985; Curtis et al., 1999), al mismo tiempo que se ha documentado una expansión de las sabanas circundantes y una disminución de la precipitación en la cuenca de Cariaco, debido a un aumento de la intensidad de los ciclos de El Niño-La Niña (ENSO) (Haug et al., 2001; Tedesco \& Thunell, 2003). Una expansión similar de las sabanas se ha registrado también en otros sondeos de la GS (Rull, 1991, 1992), de Colombia (Behling \& Hooghiemstra, 1998a) y de Guyana (Fig. 1) (Wymstra \& Van der Hammen, 1966). La hipótesis climática es que la ITZC, y con ella el cinturón lluvioso ecuatorial, se habría desplazado de nuevo hacia el sur (Curtis et al., 1999; Haug et al., 2001).

\subsection{0 años cal BP hasta el presente (Zona SM-III)}

El cambio de vegetación más notable de la secuencia se produce en esta zona. Las gramíneas (y por lo tanto, la sabana) siguen siendo dominantes, pero esta vez los elementos arbóreos desaparecen casi del todo de una forma simultánea, reflejando una reducción drástica de los bosques, a la vez que se establecen los morichales. Este cambio ya se había anticipado al final de la zona anterior, cuando se observa el primer gran pico de microcarbones, que coincide con el de los triletes psilados, interpretados como indicadores de incendios (Rull, 1999). A partir de este momento, los picos de Mauritia se alternan con los de gramíneas y microcarbones. A juzgar por el descenso de las algas, los niveles lacustres también parecen haber descendido ligeramente, hasta los actuales. El panorama general es de deforestación, disminución de la riqueza y la diversidad vegetal (sobre todo de taxones arbóreos), expansión de la sabana, establecimiento de morichales y aumento significativo del fuego, a escala regional. Se podría afirmar que es el principio del establecimiento de la Gran Sabana, tal como la conocemos hoy en día. Según Behling \& Hooghiemstra (1998a, 2001), esta configuración vegetal se debe a un incremento general de la intervención humana, a nivel de las sabanas neotropicales.

\section{Efectos del fuego y expansión de los morichales}

En otras áreas tropicales, se ha visto que la alta incidencia de fuegos parece crear un ambiente propicio para la expansión de la formación de sa-

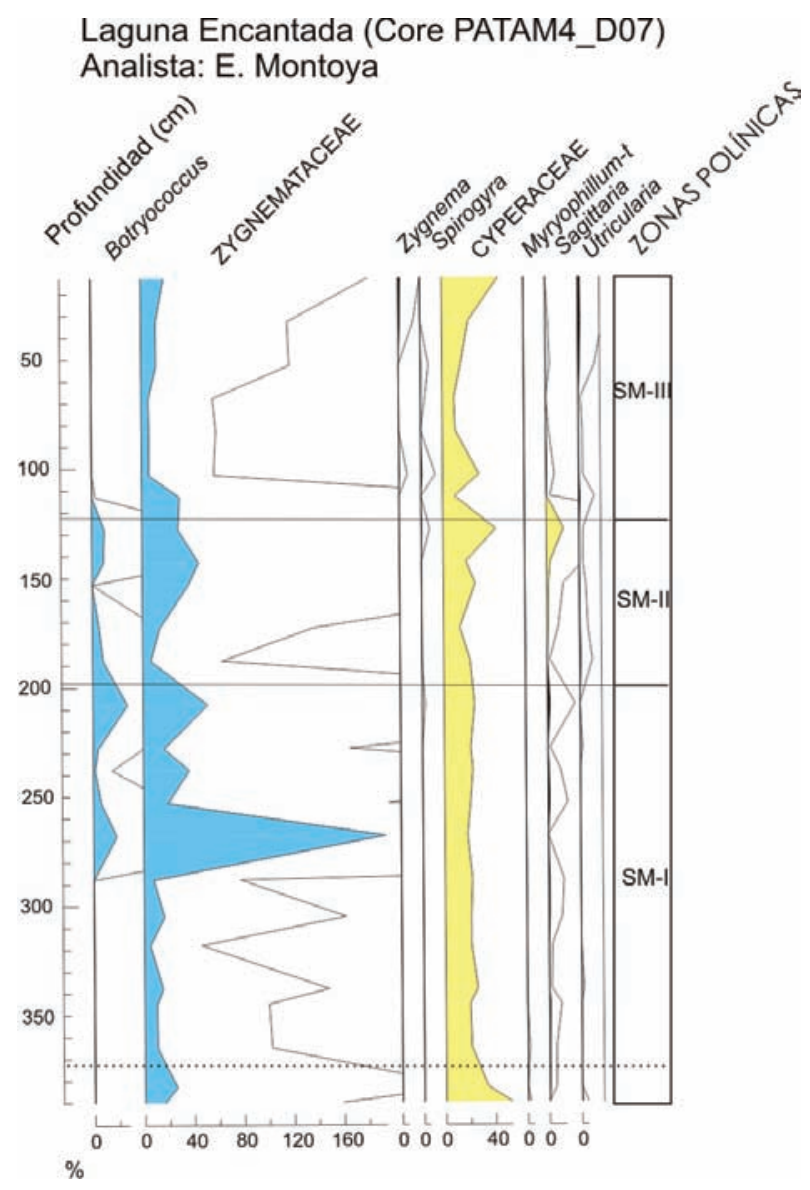

Figura 4. Diagrama polínico de taxones acuáticos y algas, expresados en porcentajes con respecto a la suma polínica.

bana, en lugares donde las condiciones climáticas y edáficas serían, en principio, más adecuadas para el desarrollo de una formación forestal (San Jose \& Fariñas, 1983; Bond et al., 2005; Bowman, 2005). Sin embargo, el presente estudio permite observar que el origen de la sabana de los alrededores de la Laguna Encantada fue muy anterior a la aparición de fuegos locales. Este hecho podría deberse a que el fuego no fuese el único factor que explique el origen de la sabana, aunque pueda favorecer su expansión. También cabe la posibilidad de que se hayan producido incendios locales anteriores al inicio de esta secuencia $(\sim 7.500$ cal BP $)$. La primera hipótesis estaría apoyada por el hecho de que en la localidad de Mapaurí (GS), situada unos $30 \mathrm{~km}$ al $\mathrm{N}$ de la Laguna Encantada, se observó la sustitución de bosques por sabanas debido a condiciones más cálidas y secas hacia los 10.000 años 


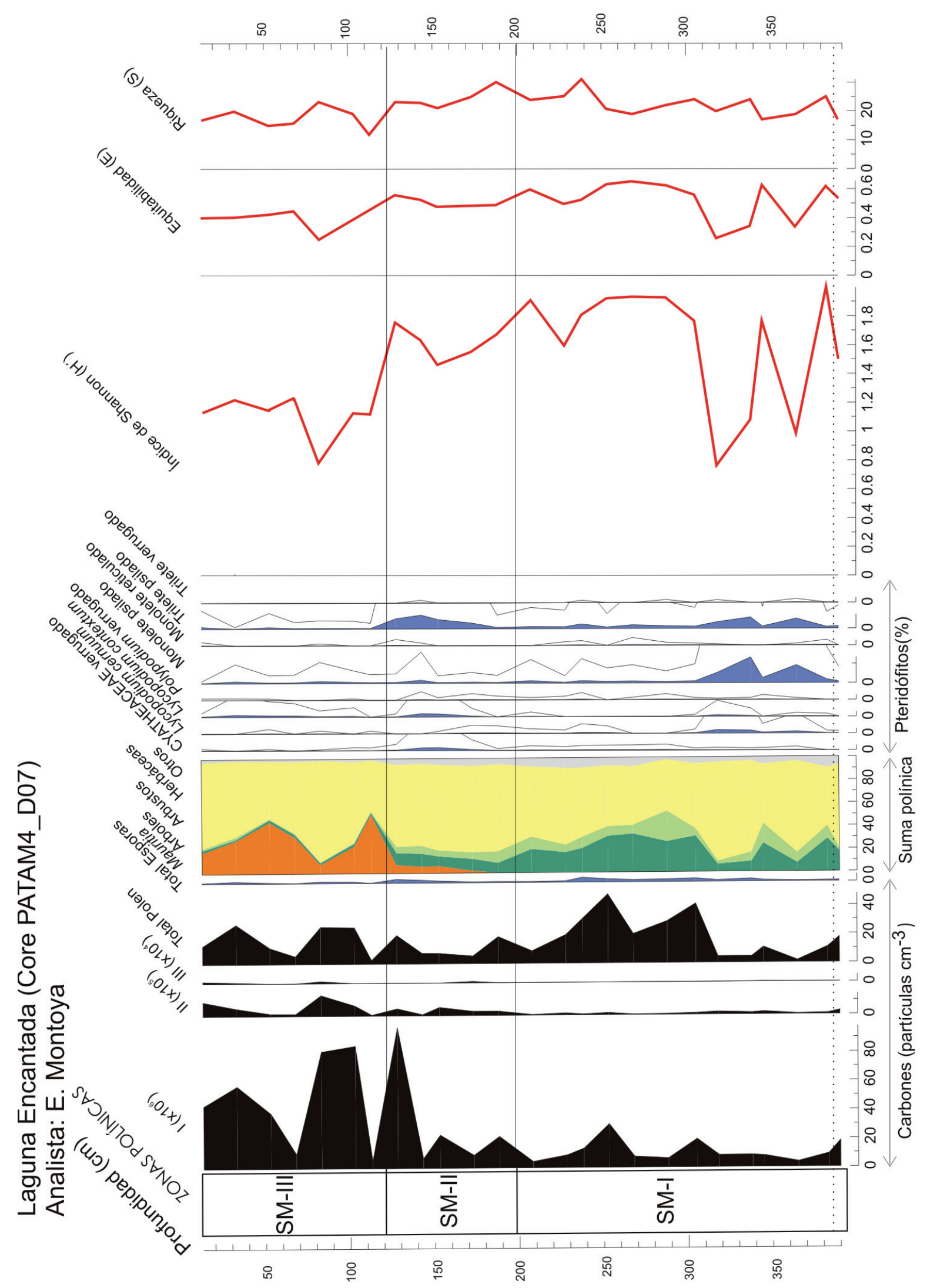

Figura 5. Diagrama de microcarbones, expresados en concentración. También se muestran las esporas de pteridófitos (en porcentaje) y los índices calculados. 


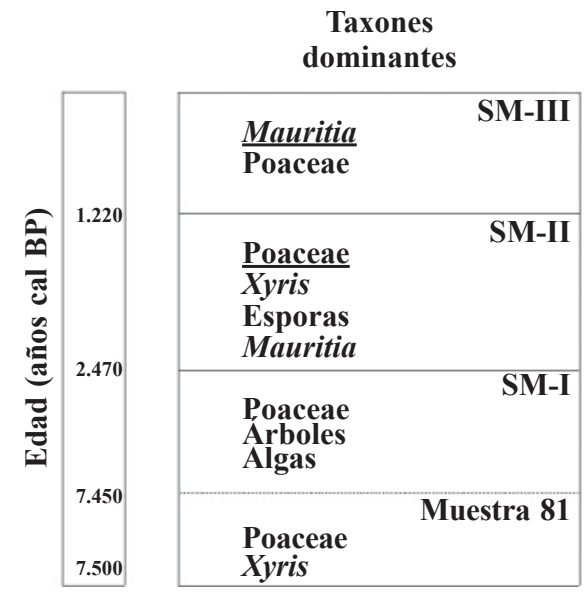

Formación dominante y clima

Sabana lisa

Morichales $\uparrow$

humedad $\sim$ actual

Saban lisa $\uparrow$

humedad $\downarrow$

Sabana (Bosque $\uparrow$ )

humedad $\uparrow$

Sabana herb-fruticosa

humedad $\downarrow$

Figura 6. Esquema resumen de la secuencia, con la zonación e interpretación del diagrama polínico.

cal BP (Rull, 2007), sin la intervención de fuegos locales. Así, a consecuencia de un aumento en las temperaturas medias de $2-3^{\circ} \mathrm{C}$, se produjo un desplazamiento altitudinal de la vegetación forestal (unos $500 \mathrm{~m}$ aproximadamente) hacia las laderas de las montañas próximas, mientras que la sabana se expandió en las áreas anteriormente ocupadas por el bosque. Con respecto a la segunda posible explicación, existen estudios donde se ha reportado la existencia de una alta correlación positiva entre la abundancia del polen de gramíneas y los microcarbones de origen regional (Rull, 2008), sugiriendo una relación entre incendios y expansión de sabanas. Sin embargo, en este estudio, se ha observado que la sabana se ha mantenido, pese a la inexistencia de fuegos locales, durante más de 5.000 años. Esto sugiere que, a pesar de poder favorecer la expansión de la sabana, el fuego no ha sido el único factor influyente en su mantenimiento, lo cual aboga por otras causas, como por ejemplo factores edáficos (Dezzeo \& Chacón, 2005). Así pues, aunque en la actualidad se reconoce al fuego como factor decisivo tanto de la expansión como del mantenimiento de la sabana (Fölster et al., 2001), los estudios paleoecológicos indican que otros factores, como el clima o las características del suelo (contenido en nutrientes, agua, etc.), pueden tener las mismas consecuencias.

En este estudio se observan las dos situaciones mencionadas anteriormente, es decir, la extensión de sabana con y sin incendios locales, con un punto de inflexión en los $\sim 1.200$ años cal BP. Antes de esa fecha, la sabana se mantenía y los bosques aumentaban o disminuían en importancia obedeciendo principalmente a cambios en el balance hídrico. Después de esa fecha, la sabana se ha mantenido en condiciones similares, pero el bosque ha desaparecido o se ha reducido drásticamente y han aparecido los morichales, a causa de un aumento notable de la frecuencia de los incendios locales. Una situación similar se observó en la localidad de Urué, a unos $40 \mathrm{~km}$ de la Laguna Encantada, donde se registraron incendios locales intensos hasta los 1.000 años BP y después prácticamente cesaron (Rull, 1999). A pesar de ello, el bosque no se recuperó, ni siquiera con la llegada de un período de clima más húmedo que, por el contrario, llevó al establecimiento de un morichal. La explicación podría estar, una vez más, en la degradación progresiva del suelo (Fölster et al., 2001; Dezzeo \& Chacón, 2005). Una conclusión extraída de la secuencia de Urué es la condición de Mauritia como colonizadora secundaria de terrenos quemados, en caso de existir la suficiente humedad como para mantener el suelo saturado de agua. El efecto del fuego consistiría básicamente en eliminar las especies de bosque potencialmente competidoras y dejar el terreno libre para los morichales (Rull, 1998a). En la Laguna Encantada, las condiciones necesarias de humedad para el establecimiento de los morichales se dieron en más de una ocasión, especialmente durante el Holoceno Medio, pero estas comunidades sólo prosperaron a partir de $\sim 1.200$ años cal BP, a pesar de la ausencia de bosques in situ. Esto sugiere que el clima no es el único condicionante y 
apoya indirectamente la hipótesis de Rull (1998a), según la cual, el área de distribución de Mauritia se habría reducido mucho durante la última glaciación, de forma que su lenta expansión postglacial no habría alcanzado la GS hasta el Holoceno Tardío.

\section{CONCLUSIONES}

La formación dominante en la zona de estudio desde el Holoceno Temprano ( $c a .7 .500$ cal BP), hasta el presente ha sido una sabana, que ha sufrido cambios en su composición taxonómica debido a variaciones en las condiciones ambientales y al régimen de incendios. Las variaciones paleoambientales y de vegetación registradas durante el Holoceno se han correlacionado con registros paleoambientales de otras áreas del norte de Sudamérica, lo que ha permitido reconocer tendencias regionales neotropicales a escala de milenios, como por ejemplo el periodo de mayor balance hídrico del Holoceno Medio ( $c a$. 4.000 cal BP), y de condiciones más secas a partir de 2.500 años cal BP. El incremento del balance hídrico del Holoceno Medio coincide con un aumento de la riqueza y la diversidad taxonómica, sobre todo en elementos de bosque. Las variaciones ambientales, principalmente los cambios en el balance hídrico, no han sido las únicas responsables de los cambios de vegetación, ya que el fuego ha ejercido un efecto de perturbación, sobre todo durante los últimos 1.200 años. Las comunidades vegetales actuales se establecieron más o menos en esa fecha, coincidiendo con la intensificación de los incendios locales. La estrecha relación entre la presencia de fuegos locales y la aparición y expansión de los morichales, observado en diversas localidades de la GS, sugiere que Mauritia podría considerarse una planta pirófila. El efecto que el fuego ha tenido sobre la vegetación difiere, según la comunidad que se considere. Así, en los bosques provoca una reducción e impide su posterior reexpansión, mientras que favorece la expansión de sabanas y morichales. En el caso de los últimos, podría afirmarse incluso que es necesario, a la vista de las evidencias recopiladas hasta el momento en la GS. La sabana, por su parte, no sólo es favorecida por el fuego, sino también por las condiciones climáticas y la degradación del suelo, sobre todo en relación a los nutrientes. Los resultados de este trabajo, junto con estudios anteriores, apoyan la hipótesis de un origen mixto, con el clima y el fuego como principales factores, de las actuales sabanas de la GS. Para una visión ecológica más precisa de los procesos implicados en los cambios de vegetación, es necesario aumentar la resolución del registro, mediante el análisis de muestras intermedias a las utilizadas en este estudio.

\section{AGRADECIMIENTOS}

Este trabajo ha sido financiado por el Ministerio de Ciencia e Innovación (anteriormente Educación y Ciencia) de España, a través del proyecto CGL-2006-00974 y la beca BES-2007-16308. Los permisos para el trabajo de campo fueron otorgados por el Ministerio de Ciencia y Tecnología (DM/0000013, 5 enero 2007, para el desarrollo de la investigación en territorio venezolano) y el Ministerio del Ambiente (IE-085, 9 febrero 2007, para la recolección de muestras) de Venezuela. Ana María Pérez se encargó de la larga y difícil tramitación de estos permisos. Aquiles y José Luis Fernández, dueños del Hato Santa Teresa, donde se encuentra la Laguna Encantada, nos ofrecieron todas las facilidades para su acceso y nos acompañaron en el trabajo de campo. Johny y Jesús, de la compañía Turi Express, nos ayudaron con el transporte de materiales. Maarten Blaauw, colaboró en el desarrollo del modelo de profundidad-edad. La revisión crítica de dos evaluadores externos, Riker Yll y Santiago Riera, contribuyó a la mejora del manuscrito.

\section{REFERENCIAS BIBLIOGRÁFICAS}

Baruch, Z. 2005. Vegetation-environment relationships and classification of the seasonal savannas in Venezuela. Flora 200: 49-64.

Beerling, D. J. \& Osborne, C. P. 2006. The origin of the savanna biome. Global Change Biol. 12: 2023-2031.

Behling, H. \& Hooghiemstra, H. 1998a. Holocene history of the Chocó rain forest from Laguna Piusbi, Southern Pacific Lowlands of Colombia. Quat. Res. 50: 300-308.

Behling, H. \& Hooghiemstra, H. 1998b. Late Quaternary palaeoecology and palaeoclimatology from pollen records of the savannas of the Llanos Orientales in Colombia. Rev. Palaeobot. Palynol. 139: 251-267.

Behling, H. \& Hooghiemstra, H. 2001. Neotropical savanna environments in space and time: Late Quaternary interhemispheric comparisons. In: Markgraf, V. (Ed.), Interhemispheric climate linkages. Academic Press, San Diego: 307-323.

Bennett, K. D. 1996. Determination of the number of zones in a biostratigraphical sequence. New Phytol. 132: 155-170.

Benninghoff, W. S. 1962. Calculation of pollen ans spore density in sediments by addition of exotic pollen in know quantities. Pollen et Spores 4: 332.

Berrío, J. C., Hooghiemstra, H., Behling, H., Botero, P. \& Van der Borg, K. 2002. Late-Quaternary savanna history of the Colombian Llanos Orientales from Lagunas Chenevo and 
Mozambique: a transect synthesis. Holocene 12: 35-48.

Birks, H. J. B. \& Birks, H. H. 1980. Quaternary Palaeoecology. Blackburn, New Jersey.

Bond, W. J., Woodward, F. I. \& Midgley, G. F. 2005. The global distribution of ecosystems in a world without fire. New Phytol. 165: 525-538.

Bowman, D. 2005. Understanding a flammable planet - climate, fire and global vegetation patterns. New Phytol. 165: 341-345.

Briceño, H. O. \& Schubert, C. 1990. Geomorphology of the Gran Sabana, Guayana Shield. Geomorphology 3: 125-141.

Bush, M. B., de Oliveira, P. E., Colinvaux, P. A., Miller, M. C. \& Moreno, J. E. 2004. Amazonian palaeoecological histories: one hill, three watersheds. Palaeogeogr. Palaeoclimatol. Palaeoecol. 214: 359-393.

Colinvaux, P. A., de Oliveira, P. E. \& Moreno, J. E. 1999. Amazon pollen manual and atlas. Harwood academic publishers, Amsterdam.

Curtis, J. H., Brenner, M. \& Hodell, D. A. 1999. Climate change in the Lake valencia Basin, Venezuela, $12600 \mathrm{yr}$ BP to present. Holocene 9: 609-619.

Dezzeo, N. \& Chacón, N. 2005. Carbon and nutrients loos in aboveground biomass along a fire induced forest-savanna gradient in the Gran Sabana, southern Venezuela. Forest Ecol. Managem. 209: 343-352.

Dezzeo, N. \& Chacón, N. 2006. Nutrient fluxes in incident rainfall, throughfall, and stemflow in adjacent primary and secondary forests of the Gran Sabana, southern Venezuela. Forest Ecol. Managem. 234: 218-226.

Dezzeo, N., Chacón, N., Sanoja, E. \& Picón, G. 2004. Changes in soil properties and vegetation characteristics along a forest-savanna gradient in southern Venezuela. Forest Ecol. Managem. 200: 183-193.

Duffin, K. I., Gillson, L. \& Willis, K. J. 2008. Testing the sensitivity of charcoal as an indicator of fire events in savanna environments: quantitative predictions of fire proximity, area and intensity. Holocene 18: 279-291.

Eden, M. J. 1974. Paleoclimatic influences and the development of savanna in southern Venezuela. J. Biogeogr. 1: 95-109.

Enache, M. D. \& Cumming, B. F. 2007. Charcoal morphotypes in lake sediments from British Columbia (Canada): an assessment of their utility for the reconstruction of past fire and precipitation. J. Paleolimnol. 38: 347-363.

Faegri, K., Kaland, P. E. \& Krzywinski, K. 1989. Textbook of pollen analysis, IV edition. Blackburn, New Jersey.

Fölster, H. 1986. Forest-savanna dynamics and desertification processes in the Gran Sabana. Interciencia (Caracas) 11: 311-316.

Fölster, H. 1992. Holocene Autochthonous Forest Degradation in Southeast Venezuela. In: Goldammer, J. G. (ed.), Tropical Forest in Transition. Birkhäuser Verlag AG, Colonia: 25-44.

Fölster, H., Dezzeo, N. \& Priess, J. A. 2001. Soil-vegetation relationship in base-deficient premontane moist forestsavanna mosaics of the Venezuelan Guayana. Geoderma 104: 95-113.

Galán, C. 1984. Memoria explicativa del mapa de zonas bioclimáticas de la cuenca del Río Caroní. C.V.G.-EDELCA, Caracas: 1-74.

Haug, G. H., Hughen, K. A., Sigman, D. M., Peterson, L. C. \& Röhl, U. 2001. Southward migration of the Intertropical
Convergence Zone through the Holocene. Science 293: 1304-1308.

Hammond, D. S., Ter Steege, H. \& Van der Borg, K. 2006. Upland Soil Charcoal in the Wet Tropical Forests of Central Guyana. Biotropica 39: 153-160.

Herrera, L. F. \& Urrego, L. E. 1996. Atlas de polen de plantas útiles y cultivadas de la Amazonia colombiana. Tropenbos, Colombia.

Hooghiemstra, H. 1984. Vegetational and climatic history of the High Plain of Bogotá, Colombia: a continuous record of the last 3.5 million years. J.Cramer, Germany.

Huber, O. 1986. La vegetación de la cuenca del Río Caroní. Interciencia (Caracas) 11: 301-310.

Huber, O. 1987. Neotropical savannas: Their flora and vegetation. TRENDS Ecol. Evol. 2: 67-71.

Huber, O. 1995a. Geophisical and Phisical Features. In: Steyermark, J. A., Berry, P. E. \& Holst, B. K. (Eds.), Flora of the Venezuelan Guayana 1: Introduction. Missouri Botanical Garden, Missouri: 1-62.

Huber, O. 1995b. Conservation of the Venezuelan Guayana. In: Steyermark, J. A., Berry, P. E. \& Holst, B. K. (Eds.), Flora of the Venezuelan Guayana 1: Introduction. Missouri Botanical Garden, Missouri: 193-218.

Huber, O. 1995c. Vegetation. In: Steyermark, J. A., Berry, P. E. \& Holst, B. K. (Eds.), Flora of the Venezuelan Guayana 1: Introduction. Missouri Botanical Garden, Missouri: 97-160.

Huber, O. 2006. Herbaceous ecosystems on the Guayana Shield, a regional overview. J. Biogeogr. 33: 464-475.

Huber, O. \& Febres, G. (Eds.). 2000. Guía ecológica de la Gran Sabana. The Nature Conservancy, Caracas.

Hurlbert, S. H. 1971. The nonconcept of species diversity: a critique and alternative parameters. Ecology 52: 577-586.

Jowsey, P. C. 1966. An improved peat sampler. New Phytol. 65: $245-248$.

Kangur, M. 2002. Methodological and practical aspects of the presentation and interpretation of microscopic charcoal data from lake sediments. Veg. Hist. \& Archaebot. 11: 289-294.

Leyden, B. W. 1985. Late Quaternary Aridity and Holocene Moisture Fluctuations in the Lake Valencia Basin, Venezuela. Ecology 66: 1279-1295.

Marchant, R. \& Hooghiemstra, H. 2004. Rapid environmental change in African and South American tropics around 4000 years before present: a review. Earth-Sci. Rev. 66: 217-260.

Moore, P. D., Webb, J. A. \& Collinson, M. E. 1991. Pollen Analysis. Blackwell Scientific Publications, Oxford.

Pielou, E. C. 1966. Species-Diversity and Pattern-Diversity in the Study of Ecological Succession. J. Theor. Biol. 10: 370-383.

Ramírez, N., Dezzeo, N. \& Chacón, N. 2007. Floristic composition, plant species abundance, and soil properties of montane savannas in the Gran Sabana, next term Venezuela. Flora 202: 316-327.

Roubik, D. W. \& Moreno, J. E. 1991. Pollen and spores of Barro Colorado Island. Missouri Botanical Garden, Missouri.

Rull, V. 1987. A note on pollen counting in palaeoecology. Pollen et Spores 29: 471-480.

Rull, V. 1991. Contribución a la paleoecología de Pantepui y la Gran Sabana (Guayana Venezolana): clima, biogeografía y ecología. Instituto Venezolano de Investigaciones 
Científicas (IVIC), Caracas.

Rull, V. 1992. Successional patterns of the Gran Sabana (southeastern Venezuela) vegetation during the last 5000 years, and its responses to climatic fluctuations and fire. $J$. Biogeogr. 19: 329-338.

Rull, V. 1998a. Biogeographical and evolutionary considerations on Mauritia (Arecaceae), based on palynological evidence. Rev. Palaeobot. Palynol. 100: 109-122.

Rull, V. 1998b. Contribución a la morfología polínica de las Urticales de los Andes venezolanos. Acta Bot. Venez. 21: 43-73.

Rull, V. 1999. A palynological record of a secondary succession after fire in the Gran Sabana, Venezuela. J. Quatern. Sci. 14: 137-152.

Rull, V. 2003. An illustrated key for the identification of pollen from Pantepui and the Gran Sabana (eastern Venezuelan Guayana). Palynology 27: 95-129.

Rull, V. 2007. Holocene global warming and the origin of the Neotropical Gran Sabana in the Venezuelan Guayana. $J$. Biogeogr. 34: 279-288.

Rull, V. 2008. New palaeoecological evidence for the potential role of fire in the Gran Sabana, Venezuelan Guayana, and implications for early human occupation. Veg. Hist. \& Archaebot. 18: 219-224.

Rull, V. \& Vegas-Vilarrúbia, T. 1997. Palynomorphs other than pollen and fern spores in glacial sediments from the Venezuelan Andes. Preliminary assessment on their potential palaeoecological value. Boletín de la Sociedad Venezolana de Geólogos 23: 5-27.
Rull, V., Abbott, M. B., Vegas-Vilarrúbia, T., Bezada, M., Montoya, E., Nogué, S. \& González, C. (en prensa). Paleoenvironmental trends in Venezuela during the last glacial cycle. In: Sánchez-Villagra, M. R., Aguilera, O. \& Carlini, A. (Eds.), Urumaco and Venezuelan Palaeontology - The fossil record of the Northern Neotropics. Indiana University Press, Indiana.

San Jose, J. J. \& Fariñas, M. R. 1983. Changes in Tree Density and Species Composition in a Protected Trachypogon Savanna, Venezuela. Ecology 64: 447-453.

Servant, M., Maley, J., Turcq, B., Absy, M. L., Brenac, P., Furnier, M. \& Ledru, M. P. 1993. Tropical forest changes during the late Quaternary in African and South American lowlands. Global Planet. Change 7: 25-40.

Stockmarr, J. 1971. Tablets with spores used in absolute pollen analysis. Pollen et Spores 13: 615-621.

Tedesco, K. \& Thunell, R. 2003. High resolution tropical climate record for the last 6,000 years. Geophys. Res. Lett. 30: 1891-1894.

Tryon, A. F. \& Lugardon, B. 1991. Spores of the Pteridophyta. Surface, wall structure, and diversity based on electron microscope studies. Springer, New York.

Van der Hammen, T. \& Absy, M. L. 1994. Amazonia during the last glacial. Palaeogeogr. Palaeoclimatol. Palaeoecol. 109: 247-261.

Wolff, E. W. 2007. When is the "present"? Quatern. Sci. Rev. 26: 3023-3024.

Wymstra, T. A. \& Van der Hammen, T. 1966. Palynological data on the history of tropical savannas in northern South America. Leidse Geologische Mededelingen 38: 71-90. 